\title{
Accurate and Stable Numerical Method for Analyzing Shielding Current Density in High-Temperature Superconducting Film Containing (racks*)
}

\author{
Atsushi KAMITANI, Teruou TAKAYAMA, Ayumu SAITOH ${ }^{1)}$ and Hiroaki NAKAMURA ${ }^{2)}$ \\ Yamagata University, Yamagata 992-8510, Japan \\ 1) University of Hyogo, Hyogo 671-2280, Japan \\ ${ }^{2)}$ National Institute for Fusion Science, Gifu 509-5292, Japan
}

(Received 27 November 2011 / Accepted 23 February 2012)

\begin{abstract}
A numerical method is proposed for analyzing the shielding current density in a high-temperature superconducting (HTS) film containing cracks/holes. If an HTS film contains cracks or holes, an integral form of Faraday's law is also imposed as the boundary condition. Since the integral form can be completely incorporated into the weak form, it is regarded as the natural boundary condition. Thus, the weak form has only to be solved with the essential boundary conditions. However, the resulting numerical solution does not satisfy the integral form exactly. In order to resolve this problem, the following method is proposed: virtual voltages be applied along the surfaces of cracks and holes so as to have Faraday's law numerically satisfied. By using the proposed method, the influence of a crack on the permanent magnet method is investigated numerically.
\end{abstract}

(c) 2012 The Japan Society of Plasma Science and Nuclear Fusion Research

Keywords: critical current density, finite element method, high-temperature superconductor, integro-differential equation, Newton method

DOI: $10.1585 /$ pfr.7.2405024

\section{Introduction}

Recently, high-temperature superconductors (HTSs) have been used for numerous engineering applications: fusion magnet, energy storage system, power cable and magnetic shielding apparatus. Since the evaluation of the shielding current density is indispensable for the design of engineering applications, several numerical methods [1-3] have been so far proposed to calculate the shielding current density. However, almost all of them can be applied only to an HTS sample containing neither a hole nor a crack.

The purpose of the present study is to develop an accurate and stable method for analyzing the time-dependent shielding current density in an HTS film with cracks and holes. Moreover, the influence of a crack on the permanent magnet method $[4,5]$ is numerically investigated by means of the method.

\section{Governing Equation}

In this section, we explain the governing equation of the shielding current density $\boldsymbol{j}$ in an HTS film containing both cracks and holes. Let us first assume that an HTS film of thickness $b$ is exposed to the time-dependent magnetic field $\boldsymbol{B} / \mu_{0}$ and that it has the same cross section $\Omega$ through the thickness direction. The boundary $\partial \Omega$ of the cross section consists of not only the outer boundary $C_{0}$

author'se-mail: kamitani@yz.yamagata-u.ac.jp

*) This article is based on the presentation at the 21st International Toki Conference (ITC21).

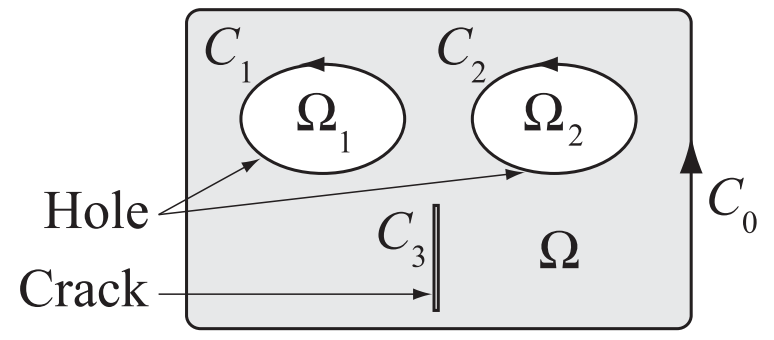

Fig. 1 A schematic view of holes and cracks in the cross section of an HTS film.

but the inner boundaries, $C_{1}, C_{2}, \cdots, C_{N}$ (see Fig. 1). Apparently, $C_{1}, C_{2}, \cdots, C_{N}$ indicate the surfaces of cracks or holes.

By taking the thickness direction as $z$-axis and choosing the centroid of the film as the origin, we use the Cartesian coordinate system $\left\langle O: \boldsymbol{e}_{x}, \boldsymbol{e}_{y}, \boldsymbol{e}_{z}\right\rangle$. In the following, $\boldsymbol{x}$ and $\boldsymbol{x}^{\prime}$ are position vectors of two points in the $x y$ plane. In addition, $\Omega_{k}$ denotes the region enclosed by $C_{k}$ and $\boldsymbol{n}$ denotes a unit normal on $\partial \Omega$.

Under the thin-plate approximation [1-3], there exists a scalar function $S(\boldsymbol{x}, t)$ such that

$$
\boldsymbol{j}=(2 / b)\left(\nabla S \times \boldsymbol{e}_{z}\right)
$$

In terms of $S(\boldsymbol{x}, t)$, the boundary condition $\boldsymbol{j} \cdot \boldsymbol{n}=0$ on $\partial \Omega$ can be written in the form, 


$$
\begin{aligned}
& S=0 \text { on } C_{0}, \\
& S=S_{k}(t) \text { on } C_{k} \quad(k=1,2, \cdots, N) .
\end{aligned}
$$

Here, $S_{k}$ 's are unknown functions of time.

The time evolution of the scalar function $S(\boldsymbol{x}, t)$ is governed by the following integro-differential equation $[2,3]$ :

$$
\mu_{0} \partial_{t}(\hat{W} S)+\boldsymbol{e}_{z} \cdot(\nabla \times \boldsymbol{E})=-\partial_{t}\left\langle\boldsymbol{B} \cdot \boldsymbol{e}_{z}\right\rangle,
$$

where \langle\rangle means an average over the thickness and $\boldsymbol{E}$ is the electric field. In addition, the operator $\hat{W}$ is defined by

$$
\begin{aligned}
\hat{W} S \equiv & \frac{2 S(\boldsymbol{x}, t)}{b} \\
& +\iint_{\Omega} Q\left(\left|\boldsymbol{x}-\boldsymbol{x}^{\prime}\right|\right) S\left(\boldsymbol{x}^{\prime}, t\right) \mathrm{d}^{2} \boldsymbol{x}^{\prime} \\
& +\sum_{l=1}^{N} \iint_{\Omega_{l}} Q\left(\left|\boldsymbol{x}-\boldsymbol{x}^{\prime}\right|\right) \mathrm{d}^{2} \boldsymbol{x}^{\prime} \cdot S_{l}(t),
\end{aligned}
$$

where the explicit form of the integration kernel $Q(r)$ is given in [3]. Note that, as compared with $\hat{W}$ for the case where an HTS film contains no holes [3], the third term in the right-hand side of (5) is added to the definition of $\hat{W}$.

As is well known, $\boldsymbol{E}$ is closely related to $\boldsymbol{j}$ in an HTS film. The relation is called the $J-E$ constitutive relation. As the relation, we adopt the power law $[2,3,6,7]$ :

$$
\boldsymbol{E}=E(|\boldsymbol{j}|) \frac{\boldsymbol{j}}{|\boldsymbol{j}|}, \quad E(j)=E_{\mathrm{C}}\left(\frac{j}{j_{\mathrm{C}}}\right)^{N},
$$

where $E_{\mathrm{C}}$ and $j_{\mathrm{C}}$ denote the critical electric field and the critical current density, respectively, and $N$ is a constant.

The initial condition to (4) is assumed as follows: $S=$ 0 at $t=0$. On the other hand, not only (2) and (3) but also the following equations are imposed as the boundary conditions to (4):

$$
\begin{aligned}
& \mu_{0} \frac{\mathrm{d}}{\mathrm{d} t} \omega_{k}[S]+\oint_{C_{k}} \boldsymbol{E} \cdot \boldsymbol{t} \mathrm{d} s=-\frac{\mathrm{d} \Phi_{k}}{\mathrm{~d} t} \\
& (k=1,2, \cdots, N) .
\end{aligned}
$$

Here, $s$ denotes an arclength along $C_{k}$ and $\Phi_{k}(t)$ is the magnetic flux linked in $\Omega_{k}$. Furthermore, the functional $\omega_{k}[S]$ is defined by

$$
\begin{aligned}
\omega_{k}[S] \equiv & \frac{2 S_{k}(t) A_{k}}{b} \\
& +\iint_{\Omega_{k}} \mathrm{~d}^{2} \boldsymbol{x} \iint_{\Omega} \mathrm{d}^{2} \boldsymbol{x}^{\prime} Q\left(\left|\boldsymbol{x}-\boldsymbol{x}^{\prime}\right|\right) S\left(\boldsymbol{x}^{\prime}, t\right) \\
& +\sum_{l=1}^{N} \iint_{\Omega_{k}} \mathrm{~d}^{2} \boldsymbol{x} \iint_{\Omega_{l}} \mathrm{~d}^{2} \boldsymbol{x}^{\prime} Q\left(\left|\boldsymbol{x}-\boldsymbol{x}^{\prime}\right|\right) \cdot S_{l}(t),
\end{aligned}
$$

where $A_{k}$ is an area of $\Omega_{k}$. Incidentally, (6) is the integral form of Faraday's law.

By solving the initial-boundary-value problem of (4), we can determine the time evolution of the scalar function $S(\boldsymbol{x}, t)$. Once $S(\boldsymbol{x}, t)$ is determined at each time, the shielding current density $\boldsymbol{j}(\boldsymbol{x}, t)$ can be easily evaluated by using (1). Apparently, the above method for calculating $\boldsymbol{j}(\boldsymbol{x}, t)$ is unaffected by the existence of cracks or holes.

\section{Virtual Voltage Method}

In this section, the numerical method for solving the initial-boundary-value problem of (4) is described in detail. In the following, the superscript $(n)$ denotes the value at time $t=n \Delta t$, where $\Delta t$ is a time-step size.

If the initial-boundary-value problem of (4) is discretized with the backward Euler method, $S^{(n)}$ becomes a solution of the following nonlinear boundary-value problem:

$$
\begin{aligned}
& \hat{G} S \equiv \mu_{0} \hat{W} S+\Delta t \boldsymbol{e}_{z} \cdot(\nabla \times \boldsymbol{E})-u=0, \\
& \gamma_{k}[S] \equiv \mu_{0} \omega_{k}[S]+\Delta t \oint_{C_{k}} \boldsymbol{E} \cdot \boldsymbol{t} \mathrm{d} s-v_{k}=0, \\
& \quad(k=1,2, \cdots, N) \\
& S \in H(\bar{\Omega}) .
\end{aligned}
$$

Here, $u=\mu_{0} \hat{W} S^{(n-1)}-\left\langle\boldsymbol{B}^{(n)}-\boldsymbol{B}^{(n-1)}\right\rangle \cdot \boldsymbol{e}_{z}$ and $v_{k}=$ $\mu_{0} \omega_{k}\left[S^{(n-1)}\right]-\left(\Phi_{k}^{(n)}-\Phi_{k}^{(n-1)}\right)$. In addition, the function space $H(\bar{\Omega})$ is defined by

$$
\begin{aligned}
H(\bar{\Omega}) \equiv & \left\{w(\boldsymbol{x}): w=0 \text { on } C_{0}\right. \\
& \left.\frac{\partial w}{\partial s}=0 \text { on } C_{k}(k=1,2, \cdots, N)\right\} .
\end{aligned}
$$

After a straightforward calculation, we can get the weak form that is equivalent to (7) and (8). It must be noted here that the derived weak form completely includes the boundary condition (8). In general, if a boundary condition is incorporated into the weak form, it is called the natural boundary condition. In this sense, (8) is the natural boundary condition though its form is considerably complicated. As is well known, the natural boundary condition is not exactly satisfied by the numerical solution. Therefore, the numerically evaluated value $N_{k}[S]$ of $\gamma_{k}[S]$ does not always vanish. In fact, the results of computations show that these tendencies become remarkable with a decrease in $b$.

In order to resolve this difficulty, we propose the virtual voltage method: a virtual voltage $\phi_{k}$ be applied along $C_{k}$ so that $N_{k}[S]$ may be exactly equal to zero. Here, $\phi_{1}, \phi_{2}, \cdots, \phi_{N}$ are all unknown constants. Specifically, the natural boundary condition (8) is replaced with the following two conditions:

$$
\begin{array}{ll}
\gamma_{k}[S]=\Delta t \phi_{k} & (k=1,2, \cdots, N), \\
N_{k}[S]=0 & (k=1,2, \cdots, N) .
\end{array}
$$

The resulting nonlinear boundary-value problem is solved with the Newton method to get $\left(S,\left\{\phi_{k}\right\}_{k=1}^{N}\right)$. At each iteration cycle of the Newton method, the linear boundaryvalue problem is solved by means of the finite element method.

\section{Numerical Simulation}

By using the above method, a numerical code has been developed for analyzing the time evolution of the shielding 
current density. In this section, we numerically reproduce the permanent magnet (PM) method $[4,5]$ by means of the code.

\subsection{PM method}

The PM method is one of the contactless methods for measuring $j_{\mathrm{C}}$. In the method, a cylindrical permanent magnet of radius $R$ and height $H$ is placed above an HTS film so that the symmetry axis of the magnet may be vertical to the film surface. The magnet is first brought closer to the film and it is subsequently moved away from the film. During the movement of the magnet, the electromagnetic force acting on the film is measured. According to the pioneering experiment by Ohshima et al. [4], the maximum repulsive force is roughly proportional to $j_{\mathrm{C}}$. This result suggests that $j_{\mathrm{C}}$ can be estimated from the measured value of the maximum repulsive force.

In the PM method, time variation of the magnetic field $\boldsymbol{B} / \mu_{0}$ is caused by moving the permanent magnet. In order to simulate the movement, the distance $L(t)$ between the magnet bottom and the film surface is assumed as follows: $L(t)=L_{\min }+\left(L_{\max }-L_{\min }\right)\left(t / \tau_{0}-1\right)\left[2 \mathrm{H}\left(t / \tau_{0}-1\right)-1\right]$. Here, $L_{\max }$ and $L_{\min }$ are the maximum and the minimum of $L(t)$, respectively, and $\tau_{0}$ denotes time at which $L(t)=L_{\min }$ is satisfied. Also, $\mathrm{H}(x)$ is Heaviside's step function.

Throughout the present study, an HTS film is assumed to have a square cross section of side length $a$. Furthermore, it is assumed to contain a crack whose cross section is a line segment connecting two points, $\left(0, \pm L_{\mathrm{c}} / 2\right)$, in the $x y$ plane. In the following, the geometrical and physical parameters are fixed as follows: $a=40 \mathrm{~mm}, b=1 \mu \mathrm{m}$, $E_{\mathrm{C}}=0.1 \mathrm{mV} / \mathrm{m}, N=20, L_{\max }=20 \mathrm{~mm}, L_{\min }=0.5 \mathrm{~mm}$, $\tau_{0}=39 \mathrm{~s}, R=2.5 \mathrm{~mm}, H=3.0 \mathrm{~mm}, B_{\mathrm{F}}=0.3 \mathrm{~T}$, and $\left(x_{\mathrm{PM}}, y_{\mathrm{PM}}\right)=(0 \mathrm{~mm}, 0 \mathrm{~mm})$. Here, $(x, y)=\left(x_{\mathrm{PM}}, y_{\mathrm{PM}}\right) \mathrm{de}-$ notes the symmetry axis of the permanent magnet. In addition, $B_{\mathrm{F}}$ is the magnitude of the magnetic flux density at $(x, y, z)=\left(x_{\mathrm{PM}}, y_{\mathrm{PM}}, b / 2\right)$ for $L(t)=L_{\mathrm{min}}$ and it is adopted as the measure of the intensity of the permanent magnet.

\subsection{Influence of crack on PM method}

Let us first derive the formula for estimating $j_{\mathrm{C}}$ by using an HTS film without any holes or cracks. After executing the numerical code with the value of $j_{\mathrm{C}}$ fixed, the electromagnetic force $F_{z}$ is evaluated. The dependence of $F_{z}$ on $L$ is numerically determined and is depicted in Fig. 2. By extrapolating the repulsive branch of the resulting $F_{z}-L$ curve, we can calculate the value of $F_{z}$ for $L=0 \mathrm{~mm}$. The value is called a maximum repulsive force and is denoted by $F_{\mathrm{M}}$.

The values of $F_{\mathrm{M}}$ are calculated for various values of $j_{\mathrm{C}}$ and are plotted in the inset of Fig. 2. This inset indicates that $j_{\mathrm{C}}$ changes in approximate proportion to $F_{\mathrm{M}}$. By applying the least-squares fitting to data points in the inset, we find $j_{\mathrm{C}} \simeq C F_{\mathrm{M}} / b \equiv f\left(F_{\mathrm{M}} / b\right)$, where $C$ is a proportionality constant. In the numerical simulation, $F_{\mathrm{M}}$ is calcu-

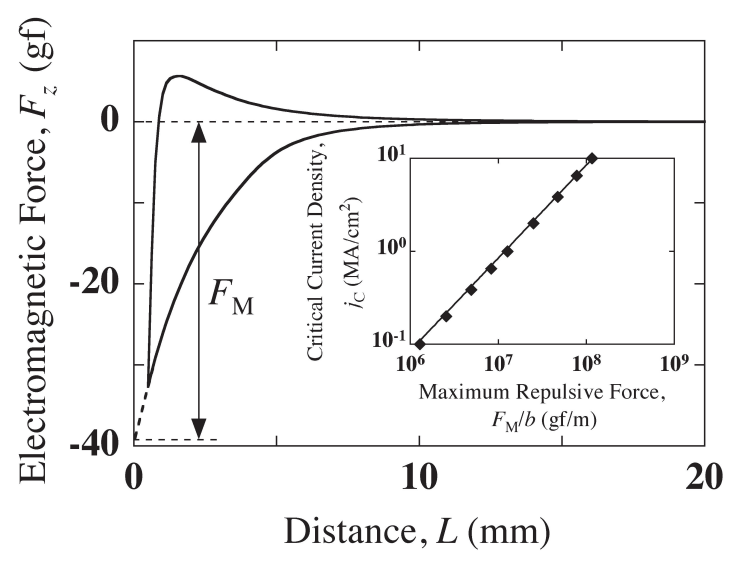

Fig. 2 Dependence of the electromagnetic force $F_{z}$ on the distance $L$ for the case with $j_{\mathrm{C}}=3.2 \mathrm{MA} / \mathrm{cm}^{2}$. The inset shows the dependence of the maximum repulsive force $F_{\mathrm{M}}$ on the critical current density $j_{\mathrm{C}}$.

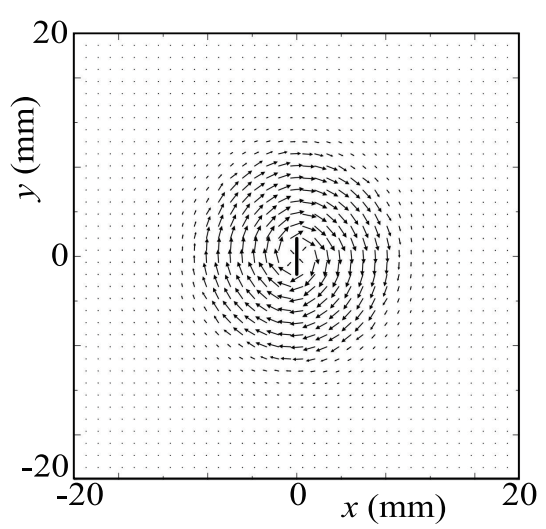

(a)

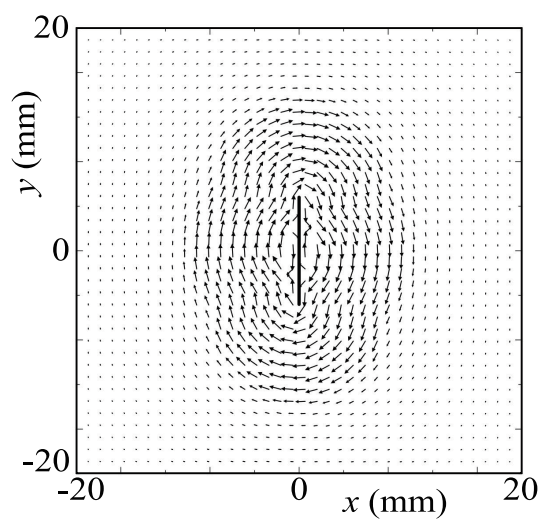

(b)

Fig. 3 Spatial distributions of the shielding current density at $t=$ $\tau_{0}$ for the case with $j_{\mathrm{C}}=3.2 \mathrm{MA} / \mathrm{cm}^{2}$. Here, (a) $L_{\mathrm{c}}=$ $3.2 \mathrm{~mm}$ and (b) $L_{\mathrm{c}}=9.6 \mathrm{~mm}$. In both figures, cracks are denoted by thick line segments.

lated and, subsequently, the estimated value $j_{\mathrm{C}}^{*}$ of the critical current density is determined by using $j_{\mathrm{C}}^{*}=f\left(F_{\mathrm{M}} / b\right)$.

As the measure of the accuracy of the PM method, we use the relative error defined by $\epsilon \equiv\left|j_{\mathrm{C}}^{*}-j_{\mathrm{C}}\right| / j_{\mathrm{C}}$. Note 


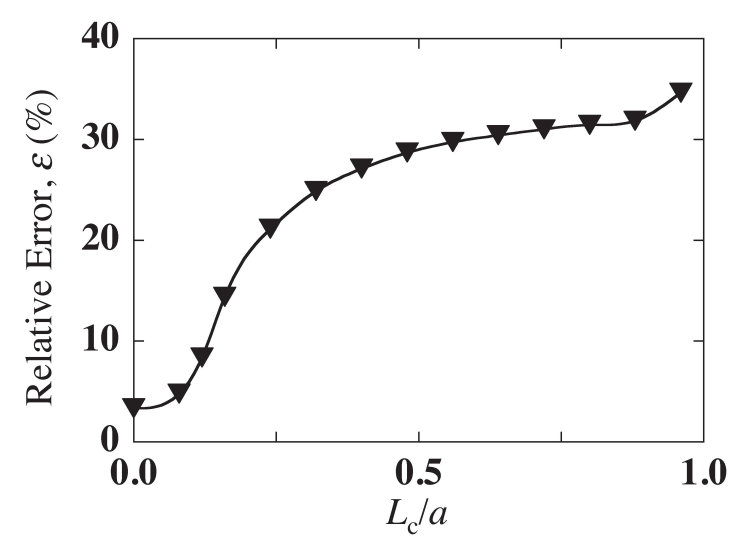

Fig. 4 Dependence of the relative error $\epsilon$ on the crack size $L_{\mathrm{c}}$ for the case with $j_{\mathrm{C}}=3.2 \mathrm{MA} / \mathrm{cm}^{2}$.

that the estimation formula $j_{\mathrm{C}}^{*}=f\left(F_{\mathrm{M}} / b\right)$ is derived for the case where an HTS film contains neither a crack nor a hole. In other words, its accuracy remains high if and only if the shielding current density is almost axisymmetric. Therefore, if the axial symmetry of the $j$-distribution is lost, the relative error $\epsilon$ increases considerably.

Next, we investigate how the PM method is affected by the crack size. To this end, the $\boldsymbol{j}$-distributions are determined for various values of the crack size $L_{\mathrm{c}}$. Typical examples of the $\boldsymbol{j}$-distributions are shown in Figs. 3 (a) and 3 (b). For the case with $L_{\mathrm{c}}=3.2 \mathrm{~mm}$, the $\boldsymbol{j}$-distribution is almost axisymmetric and, hence, it is hardly influenced by the crack. In contrast, for the case with $L_{\mathrm{c}}=9.6 \mathrm{~mm}$, it is deformed prolately so that the axial symmetry is broken. These results imply that an increase in $L_{\mathrm{c}}$ will degrade the accuracy of the PM method. In order to investigate this tendency quantitatively, the relative error $\epsilon$ is evaluated as a function of $L_{\mathrm{c}}$ and is depicted in Fig. 4. As is expected, the relative error monotonously increases with the crack size. This monotonous increase of $\epsilon$ is based on the fact that the axial symmetry of the $\boldsymbol{j}$-distribution is gradually lost with increasing crack size.

\section{Conclusion}

We have proposed an accurate and stable method for calculating the shielding current density in an HTS film containing cracks and holes. The basic idea of this method is to apply virtual voltages along the surfaces of cracks and holes so as to have Faraday's law numerically satisfied. On the basis of the proposed method, a numerical code has been developed for analyzing the time evolution of the shielding current density. As an application of the code, the influence of a crack on the PM method has been investigated numerically.

Conclusions obtained in the present study are summarized as follows: the axial symmetry of the $\boldsymbol{j}$-distribution is gradually lost with an increase in the crack size until the accuracy of the PM method is degraded remarkably.

\section{Acknowledgments}

This work was supported in part by Japan Society for the Promotion of Science under a Grant-in-Aid for Scientific Research (C) No.21560287. A part of this work was also performed with the support and under the auspices of the NIFS Collaboration Research program (NIFS11KNTS011, NIFS10KNXN178).

[1] Y. Yoshida, M. Uesaka and K. Miya, IEEE. Trans. Magn. 30, 3503 (1994).

[2] A. Kamitani, T. Takayama and S. Ikuno, IEEE. Trans. Magn. 44, 926 (2008).

[3] A. Kamitani, T. Takayama and S. Ikuno, IEEE. Trans. Magn. 47, 1138 (2011).

[4] S. Ohshima, K. Takeishi, A. Saito, M. Mukaida, Y. Takano, T. Nakamura, T. Suzuki and M. Yokoo, IEEE Trans. Appl. Supercond. 15, 2911 (2005).

[5] S. Ikuno, T. Takayama, A. Kamitani, K. Takeishi, A. Saito and S. Ohshima, IEEE Trans. Appl. Supercond. 19, 3750 (2009).

[6] R. Fresa, G. Rubinacci, S. Ventre, F. Villone and W. Zamboni, IEEE. Trans. Magn. 45, 988 (2009).

[7] G.P. Lousberg, J.F. Fagnard, M. Ausloos, P. Vanderbemden and B. Vanderheyden, IEEE Trans. Appl. Supercond. 20, 33 (2010). 